\title{
BMJ Open Longitudinal circulating placental growth factor (PIGF) and soluble FMS- like tyrosine kinase-1 (sFlt-1) concentrations during pregnancy in Asian women: a prospective cohort study
}

\author{
Qiu Ju Ng, ${ }^{\oplus}$ Jonathan Youxiang Han, ${ }^{2}$ Seyed Ehsan Saffari, ${ }^{\circ}$ \\ George Seow-Heong Yeo, ${ }^{4}$ Bernard Su Min Chern, ${ }^{5}$ Kok Hian Tan ${ }^{5}$
}

To cite: $\mathrm{Ng}$ QJ, Han JY, Saffari SE, et al. Longitudinal circulating placental growth factor (PIGF) and soluble FMSlike tyrosine kinase-1 (sFlt-1) concentrations during pregnancy in Asian women: a prospective cohort study. BMJ Open 2019;9:e028321. doi:10.1136/ bmjopen-2018-028321

\section{- Prepublication history for} this paper is available online. To view these files please visit the journal online (http://dx.doi. org/10.1136/bmjopen-2018028321).

Received 12 December 2018 Revised 29 April 2019 Accepted 29 April 2019
D) Check for updates

(C) Author(s) (or their employer(s)) 2019. Re-use permitted under CC BY-NC. No commercial re-use. See rights and permissions. Published by BMJ.

For numbered affiliations see end of article.

Correspondence to

Dr Qiu Ju Ng;

qiuju89@gmail.com

\section{ABSTRACT}

Objectives To analyse the placental growth factor (PIGF) and soluble FMS-like tyrosine kinase-1 (sFlt-1) trends in the normal pregnant Asian population in Singapore.

Design A prospective study was conducted.

Setting The largest tertiary hospital in Singapore.

Methods Women with single viable pregnancies, less than 14 weeks of gestation, were recruited between September 2010 and November 2013 in KK Women's and Children's Hospital. They were followed up from recruitment till their postnatal discharge from the hospital. There were four antenatal visits: gestational age (GA) less than $14+0$ weeks of gestation (V1), GA 18+0 to 22+0 weeks (V2), GA $28+0$ to $32+0$ weeks (V3) and GA 34+0 and above (V4). Serum biochemical markers (sFIt-1, PIGF) were measured at each visit.

Results There were 934 participants in the study, of which 674 had normal pregnancy outcomes. The sFlt-1 remained relatively constant till GA 28-32 weeks before it increased $(p<0.001)$. The sFlt- 1 levels increased earlier before 30 weeks' of gestation among the Malay participants and the other ethnicities. For PIGF, the levels increased from the first to the third trimester, peaking at $30-32$ weeks before decreasing $(p<0.001)$. Its serum levels significantly differed among the Indian participants and other ethnicities as compared with the Malay and Chinese participants at V3 and V4, $(p=0.04$ and $\mathrm{p}<0.001$, respectively).

Conclusion There are significant differences in the PIGF and sFlt-1 concentrations during pregnancy between different ethnicities, which should be taken into consideration when using these references values for further research.

\section{INTRODUCTION}

Pre-eclampsia is one of the leading causes of maternal and foetal morbidity and mortality and is estimated to occur in about $4 \%$ of pregnancies $\quad$ worldwide. ${ }^{1} \quad$ Pre-eclampsia is a multi-systemic disease and the exact

\section{Strengths and limitations of this study}

- One of the strengths of our study was the large sample size. To the best of our knowledge, this is one of the few studies done in Asia which looked at the trend of soluble FMS-like tyrosine kinase-1 (sFIt-1) and placental growth factor (PIGF) in normal pregnancies.

- This was a prospective cohort study which recruited women at our low-risk obstetrics clinic. Thus, our results would be relevant to the low-risk obstetrics population.

- However, the number of subjects in some ethnic groups was small in our study, which may affect the precision of our finding.

- Our study collected the blood samples at different gestational weeks, which might have had an impact on the timing of the peak of sFlt-1 or the drop in PIGF.

- Finally, the observed differences among ethnic groups might be due to uncontrolled confounders rather than genetic variation. Thus, interpretation of our finding needs caution.

pathophysiology is still unclear. Numerous studies indicate that the imbalance between placental pro-angiogenic and antiangiogenic vasoactive agents, such as placental growth factor (PIGF) and soluble FMS-like tyrosine kinase 1 (sFlt-1), respectively, contributes to the pathogenesis of pre-eclampsia. ${ }^{23}$

PIGF is a member of the vascular endothelial growth factor (VEGF) family which is primarily synthesised in the placenta ${ }^{4}$ and binds to the VEGRF-1 receptor, resulting in angiogenic activity. sFlt-1 is a natural antagonist to VEGF and PIGF by binding to them and preventing them from interacting with their receptors, resulting in anti-angiogenic 
activity and systemic endothelial dysfunction. Multiple studies have found that gestational changes in sFlt-1 and PIGF correlate with the onset and severity of pre-eclampsia ${ }^{34}$ and the sFlt-1/PlGF ratio has been investigated as a predictive biomarker for pre-eclampsia..$^{5-10}$

It would be essential to know the trend of plasma sFlt-1 and PIGF biomarkers in normal pregnancies and the normal reference values so as to be able to validate the use of cutoffs of sFlt-1, PlGF and the sFlt-1/PlGF ratio for the prediction of pre-eclampsia. Interestingly, there are few longitudinal studies done to investigate these biomarkers in normal pregnancies ${ }^{8}{ }^{11-14}$ and most of these studies are conducted in non-Asian countries with a mainly Caucasian population. In view of the lack of data among the Asian population, our study aims to analyse the sFlt- 1 and PlGF trends in the normal pregnant Asian women in Singapore.

\section{METHODS}

\section{Patient and public involvement}

The participants could obtain the study results from the clinical coordinators. Patients and the public were not involved in the design or planning of the study.

\section{Participants}

This study is a part of the Neonatal and Obstetrics Risks Assessment (NORA) prospective cohort study, which focused on evaluating the use of clinical, biochemical and biophysical markers to predict a risk of adverse pregnancy outcomes, including pre-eclampsia so that early clinical intervention can be implemented. NORA was conducted in KK Women's and Children's Hospital $(\mathrm{KKH})$, the largest tertiary maternity hospital in Singapore that manages the largest number of complicated pregnancies locally. Women who had viable, singleton pregnancies and were attending their first antenatal visit, at less than $14+0$ weeks of gestation, were screened for eligibility for the study in KKH between September 2010 and November 2013. The exclusion criteria were multiple gestations, chronic medical conditions such as renal disease or systemic lupus erythematosus and pregnancies complicated by aneuploidy or foetal anomaly. Written informed consent was obtained from each participant. The women recruited consisted of four different ethnicities; Chinese, Malay, Indian and others.

A total of 3271 women were screened and only 2820 women were eligible and out of which, 1013 women consented to participate in the study. Out of the 1013 participants, $934(92.2 \%)$ patients completed all four

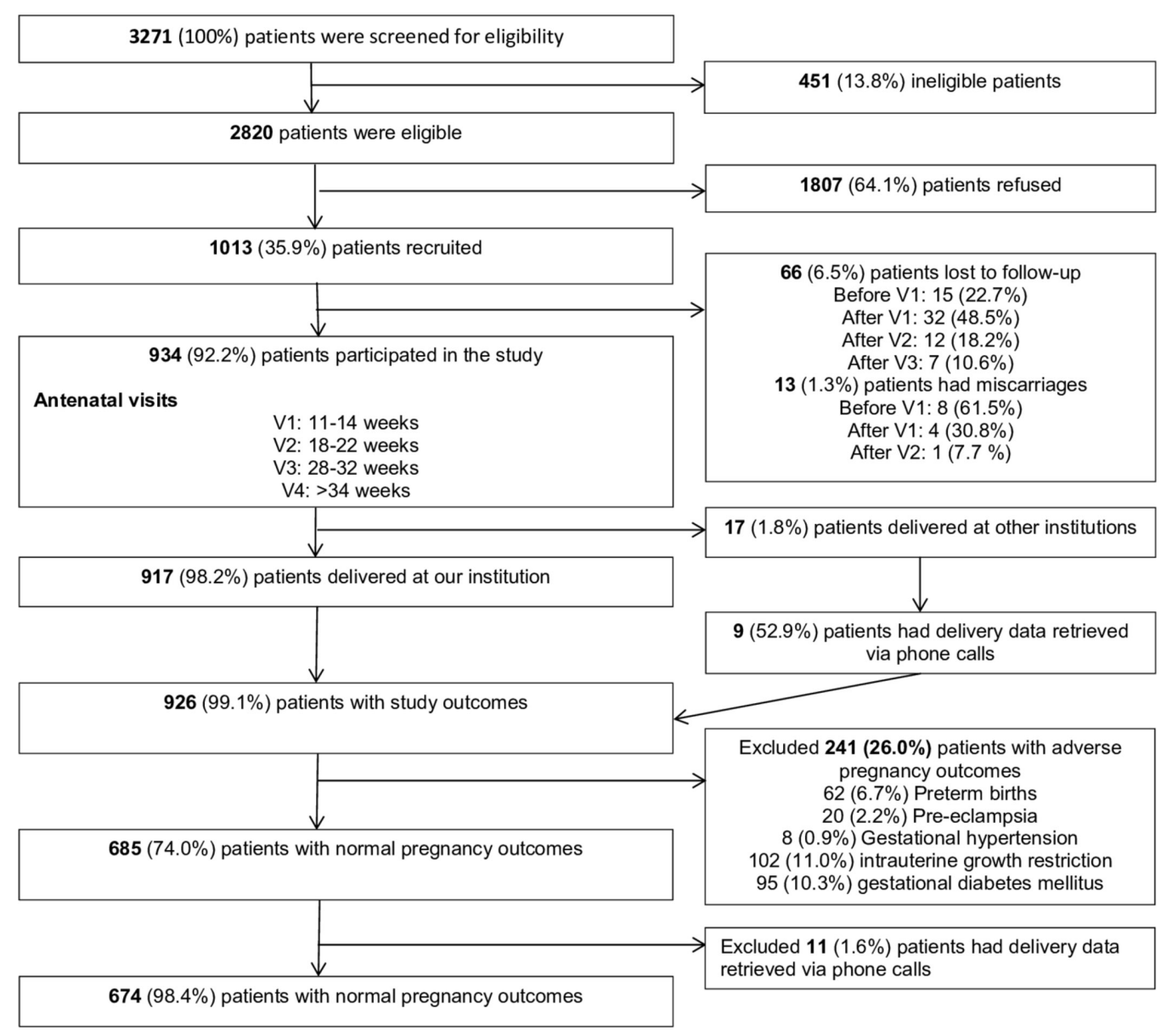

Figure 1 Neonatal and Obstetrics Risks Assessment study recruitment. 
antenatal visits. As eight participants did not deliver in our institution, we studied the outcomes of the remaining $926(99.0 \%)$ patients (figure 1$)$.

Of the 926 women, there were $241(26.0 \%)$ women with adverse pregnancy outcomes who were excluded from the current analysis. These adverse pregnancy outcomes, which were not mutually exclusive, consisted of $62(6.7 \%)$ preterm births defined as gestational age (GA) $<37+0$ weeks, $20(2.2 \%)$ pre-eclampsia, $8(0.9 \%)$ gestational hypertension, $102(11.0 \%)$ intrauterine growth restriction (IUGR), defined as estimated foetal weight or abdominal circumference less than the fifth percentile adjusting for gender and ethnicity, or birth weight less than the third percentile, and $95(10.3 \%)$ gestational diabetes mellitus. Of the $685(74.0 \%)$ women with normal pregnancy outcomes, we further excluded those with pre-existing hypertension or diabetes, which may affect the sFlt- 1 and PlGF values. Hence, we analysed the sFlt- 1 and PlGF trends in the remaining 674 (72.8\%) women with normal pregnancy outcomes.

The women were closely followed up throughout their pregnancies and clinical and laboratory data were collected prospectively. On recruitment at 11-14 weeks (V1), an interview and a dating ultrasound scan were completed, and blood samples were collected. Subsequently, the women were seen at 18-22 weeks, 28-32 weeks and 34 weeks and above. The remaining visits were defined as V2, V3 and V4, respectively. The women had routine ultrasound investigations at 18-22 weeks for screening of foetal anomalies and at 28-32 weeks for growth of the fetus. In addition, Doppler studies on umbilical, fetal middle cerebral and uterine arteries were performed at 18-22 weeks, 28-32 weeks and 34 weeks and above for detection of IUGR. Following delivery, data collection included the details and mode of delivery and neonatal outcomes.

\section{Sample collection and assays}

Serum levels of sFlt-1 and PIGF were measured at all study visits. For the serum samples, $5-10 \mathrm{~mL}$ venous blood was collected in non-heparinised tubes (serum) and assayed using the Elecsys 2010 and Cobas e411 electrochemiluminescence immunoassay machine. The detection limits were approximately $6 \mathrm{pg} / \mathrm{mL}$ for sFlt- 1 and $<2 \mathrm{pg} / \mathrm{mL}$ for PIGF.

\section{Statistical analysis}

The serum biomarkers-sFlt-1 and PlGF-and the sFlt-1/ PIGF ratio at each visit were compared among the four ethnic groups using Kruskal Wallis test followed by post-hoc analysis of pairwise tests adjusted for multiple testing. One-sample t-test method was used to check whether change in sFlt-1 over the last two visits was significant. We performed the one-way analysis of variance analysis to investigate the trend of PlGF over the first three visits. Statistical analysis was performed using SAS V.9.4 for Windows. Statistical significance was set at $\mathrm{p}<0.05$. We adjusted the difference in biomarkers for possible

\begin{tabular}{lcc}
\hline \multicolumn{3}{l}{ Table 1 Characteristics of the study cohort } \\
\hline Demographics & $\mathbf{N}$ & Mean \pm SD/\% \\
\hline Age (years) & 674 & $30.5 \pm 1.0$ \\
\hline Ethinicity & 344 & 51.0 \\
\hline Chinese & 200 & 29.7 \\
Malay & 74 & 11.0 \\
Indian & 56 & 8.3 \\
\hline Other & & \\
\hline Parity (excluding current pregnancy) & 358 & 53.1 \\
\hline Nullip & 217 & 32.2 \\
\hline Para 1 & 99 & 14.7 \\
\hline Para 2 and above & 674 & $23.8 \pm 4.4$ \\
\hline BMl at booking (kg/m ${ }^{2}$ ) & 674 & $39.0 \pm 1.0$ \\
\hline GA at delivery (weeks) & 674 & $3213.2 \pm 0.34$ \\
\hline Birth weight at delivery (grams) & & \\
\hline
\end{tabular}

confounders, which are parity, body mass index (BMI) and estimated foetal weight at visits V1-V3.

\section{RESULTS}

The demographics of the population are described in table 1 . The mean age was $30.5( \pm 1.0)$ years old and half of the patients $(53.1 \%)$ were primiparous. The mean BMI at booking was $23.8( \pm 4.4) \mathrm{kg} / \mathrm{m}^{2}$ and the mean GA at booking was $39.0( \pm 1.0)$ weeks' of gestation. In terms of ethnicity, half of them were Chinese participants (51.0\%) while $29.7 \%$ were Malay participants, $11.0 \%$ were Indian participants and the remaining $8.3 \%$ were of other ethnicity such as Eurasians.

The changes in sFlt- 1 and PIGF in pregnancy are shown in figures 2 and 3, classified based on ethnicity. The mean and median values of the plasma biomarkers at each visit are shown in table 2. For sFlt-1, the plasma levels remained relatively constant until about 28-32 weeks of

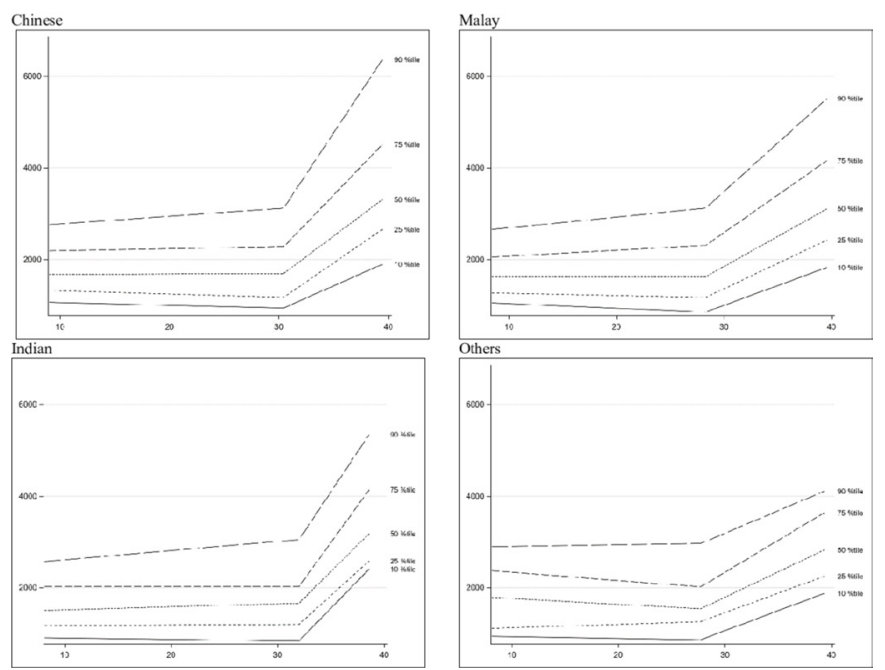

Figure 2 Soluble FMS-like tyrosine kinase- 1 trend across gestational age. 

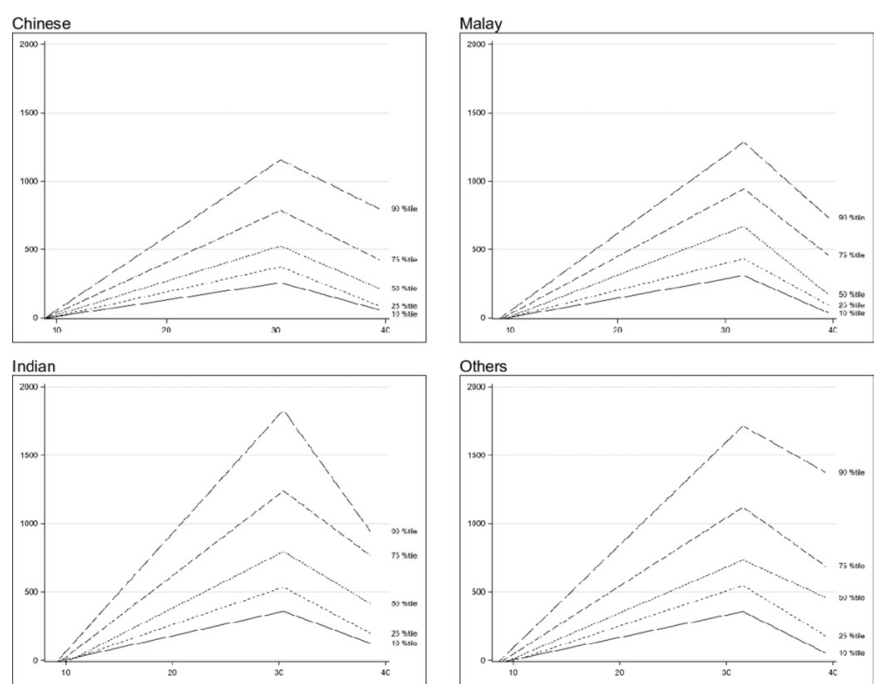

Figure 3 Placental growth factor trend across gestational age.

gestation and increased significantly in the third trimester $(p<0.001)$ across all four ethnic groups. The overall median values (10th-90th percentile) of sFlt-1 at V1, V2, $\mathrm{V} 3$ and V4 were $1600 \mathrm{pg} / \mathrm{mL}$ (996.2-2666), $1752.0 \mathrm{pg} /$ $\mathrm{mL}(941.7-3259.0), 1690.5 \mathrm{pg} / \mathrm{mL}(941.2-2984.5)$ and $2640.0 \mathrm{pg} / \mathrm{mL}$ (1550.0-4820.0), respectively. The sFlt-1 levels started to rise significantly before 30 weeks' of gestation among the Malay participants and the other ethnicities as compared with the Chinese and Indian participants whose levels only increased after 30 weeks' of gestation (figure 2). There were no significant differences in the sFlt-1 values between the different ethnicities at each of the visits (table 2).

For PlGF, the levels increased from the first to the third trimester, peaking at 30-32 weeks before decreasing $(\mathrm{p}<0.001)$. The overall median values $(10 \mathrm{th}-90$ th percentile) of PlGF at V1, V2, V3 and V4 were $34.7 \mathrm{pg} / \mathrm{mL}$ (20.3-70.3), $263.0 \mathrm{pg} / \mathrm{mL}(148.8-474.4), 636.4 \mathrm{pg} / \mathrm{mL}$ (257.2-1405.5) and $369.9 \mathrm{pg} / \mathrm{mL}(130.1-1077.0)$, respectively. The PIGF levels dropped at a later gestation among the Malay and other ethnicities participants as compared with the Chinese and Indian participants whose levels peaked at 30 weeks. There were significant differences in the PlGF values between the different ethnicities where the values are significantly higher among the Indian and other ethnicities participants as compared with the Malay and Chinese participants at V3 and V4, $\mathrm{p}=0.04$ and $\mathrm{p}<0.001$, respectively) (table 2 ).

The sFlt-1/PlGF ratios are shown in table 2 . The ratios reflected a decrease from $\mathrm{V} 1$ to $\mathrm{V} 3$ and increases between V3 and V4 (figure 4). The values were also significantly different among the four ethnic groups at V1, V2 and V4.

\section{DISCUSSION}

\section{Main findings}

This study describes the changes in sFlt-1 and PlGF levels throughout pregnancy in an Asian population with normal outcomes in Singapore and provides the references ranges for the 10th-90th percentiles. From the longitudinal studies done in other countries, ${ }^{6} 8911-13$ the sFlt-1 level is almost ten times that of a non-pregnant woman and stays constant until the last 2 months of pregnancy when it increases. Our results were consistent with the other studies as it showed that sFlt-1 remained relatively stable till GA 28-32 weeks before it started to rise. Interestingly, there was a significant difference in the rise of sFlt-1 levels among the four ethnic groups. Since the sFlt-1 levels rise before $30+0$ weeks of gestation among the Malay and other ethnicities participants, it would be important to correct for ethnicity when using these reference values to obtain a cut-off for prediction of preeclampsia. Wolf et al investigated the difference in levels of sFlt-1 between a woman's first and second pregnancies and found that Hispanic women had higher levels than white women in the first pregnancy but lower levels in the subsequent pregnancies. ${ }^{15}$ Hence, there could be a genetic component that affects the sFlt- 1 level.

For PlGF, its plasma level is about twice that of a non-pregnant woman in the first trimester and increases up to 32-34 weeks before decreasing in the last 2 months of pregnancy. ${ }^{68911-14}$ Our results on PlGF trend were also consistent with what has been observed where PlGF increases steadily to a peak at about 30-32 weeks before decreasing. There were significant differences in values among the four ethnic groups at V2, V3 and V4 ( $<<0.001)$ where the Chinese and Malay participants have significantly lower values than the Indian and other ethnicities participants. This has been reflected in other studies which showed that PlGF values differ between the various ethnic groups. ${ }^{15-18}$

\section{Strengths and limitations}

One of the strengths of our study was the large sample size. To the best of our knowledge, this is one of the few studies done in Asia which looked at the trend of sFlt-1 and PlGF in normal pregnancies. In addition, this was a prospective cohort study which recruited women at our low-risk obstetrics clinic. Thus, our results would be relevant to the low-risk obstetrics population. However, the number of subjects in some ethnic groups was small in our study, which may affect the precision of our finding. Also, our study collected blood samples at different GA windows, and the timing of specimen collection might have had an impact on the timing of the peak of sFlt-1 or the drop in PlGF. Finally, the observed differences among ethnic groups might be due to uncontrolled confounders rather than genetic variation. Thus, interpretation of our finding needs caution.

\section{Interpretation}

The changes in sFlt-1 and PlGF during pregnancy found in our study were consistent with that in other populations. We can also use these values in our local population to investigate their role in predicting pre-eclampsia and other possible complications such as IUGR. 


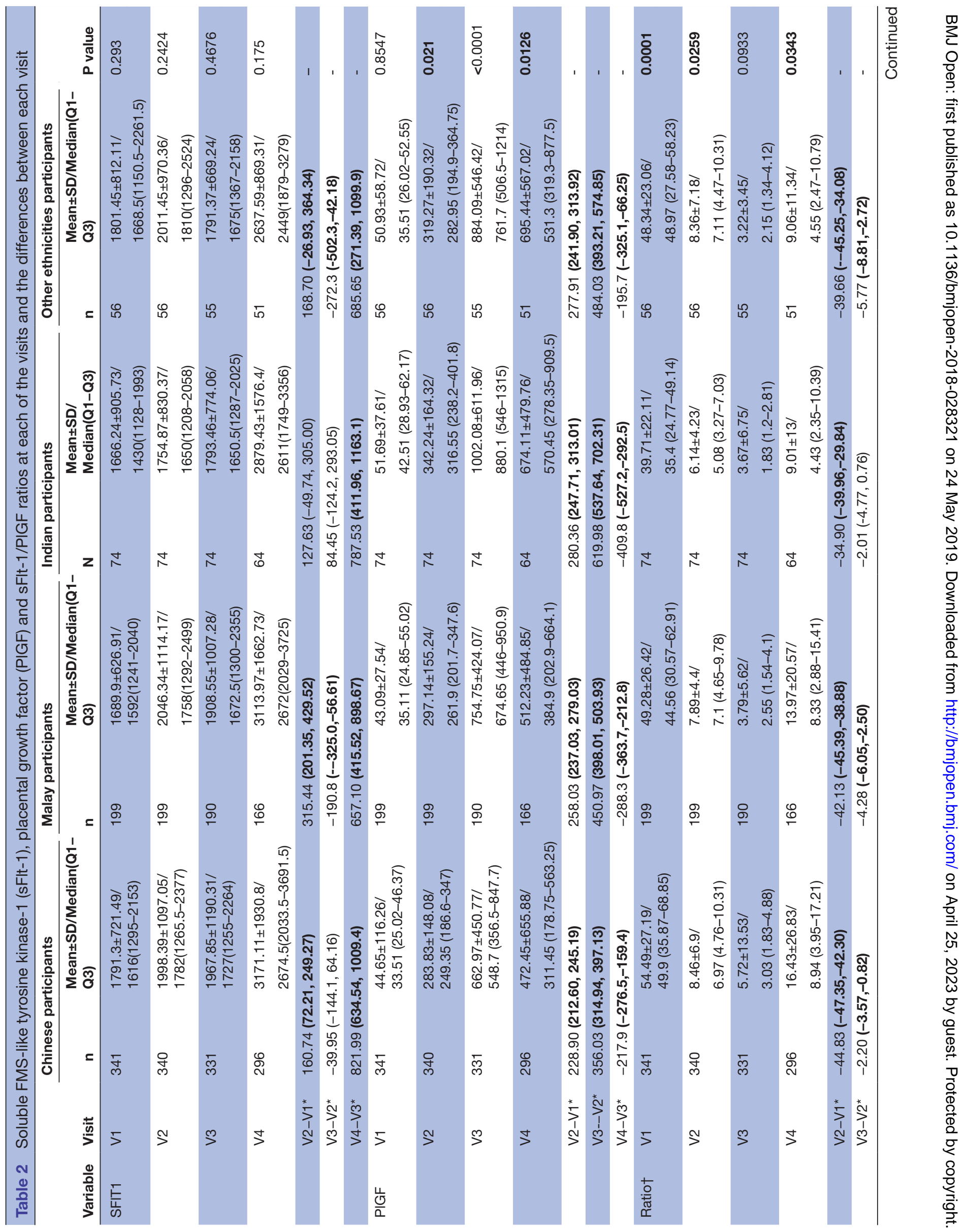



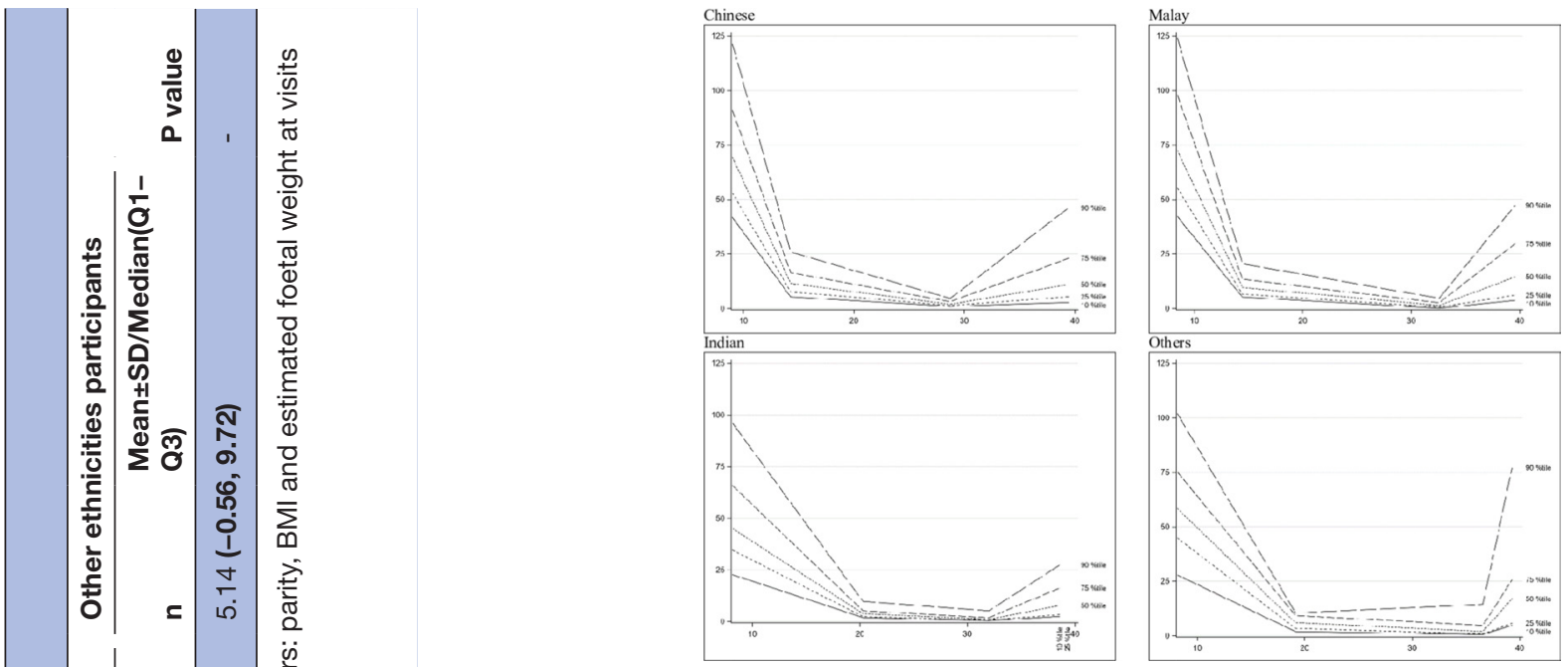

Figure 4 Ratio trend across gestational age.

\section{CONCLUSION}

The trends in PlGF and sFlt-1 concentrations over gestation in Asian women in Singapore are consistent with the findings in other studies. As there are significant differences in the biomarkers between different ethnicity, ethnicity should be an important factor in the analysis of these biomarkers. Further research would encompass using these reference values to compare with women who developed pre-eclampsia and obtaining a predictive cut-off ratio for the risk of pre-eclampsia.

\section{Author affiliations}

${ }^{1}$ KK Women's and Children's Hospital, Singapore, Singapore

${ }^{2}$ Department of Obstetrics and Gynaecology, KK Women's and Children's Hospital, Singapore

${ }^{3}$ Centre for Quantitative Medicine, Duke—NUS Medical Centre, Singapore, Singapore

${ }^{4}$ Department of Maternal-Fetal Medicine, KK Women's and Children's Hospital, Singapore

${ }^{5}$ KK Women's and Children's Hospital, Division of Obstetrics and Gyneacology, Singapore, Singapore

Acknowledgements The authors would like to acknowledge the help of $\mathrm{Mr} \mathrm{Ng}$ Mor Jack, Ms Tey Wan Shi and Ms Nyo Mie Win (supported by the hospital centre grant-Integrated Platform for Research in Advancing Metabolic Health Outcomes of Women and Children, IPRAMHO/CGAug16C008). They would also like to acknowledge Prof John Carson Ellen, Prof Jim Zhang and Prof Kenneth Yung Chiang Kwek for their advice regarding data and epidemiological analysis.

Contributors KHT was the leader of the project and designed the study with QJN, JYH, SES, GS-HY and BC. QJN, JYH, SES and KHT did literature review, data collection and analysis of the results. KHT, QJN and SES drafted the manuscript. GS-HY and BC supervised the data analysis and were responsible for the editing of the manuscript. All of the authors critically reviewed the manuscript before publication.

Funding The NORA study was funded by the National Medical Research Council (Project Grant number NMRC/PPG/KKH/2010). The National Medical Research Council Programme Project Grant was a competitive grant where the study was subjected to peer review before being awarded with the grant. There was no patient or public panel involved in the review of the grant and the National Medical Research Council did not play any part in conducting the research or writing of the manuscript.

Competing interests None declared.

Patient consent for publication Not required. 
Ethics approval The NORA study was approved by theEthics Committee and funded by the National Medical Research Council.

Provenance and peer review Not commissioned; externally peer reviewed.

Data sharing statement The data that support the findings of this study area available from the corresponding 3 author, $\mathrm{Ng} \mathrm{QJ}$, upon reasonable request.

Open access This is an open access article distributed in accordance with the Creative Commons Attribution Non Commercial (CC BY-NC 4.0) license, which permits others to distribute, remix, adapt, build upon this work non-commercially, and license their derivative works on different terms, provided the original work is properly cited, appropriate credit is given, any changes made indicated, and the use is non-commercial. See: http://creativecommons.org/licenses/by-nc/4.0/.

\section{REFERENCES}

1. Abalos E, Cuesta C, Grosso AL, et al. Global and regional estimates of preeclampsia and eclampsia: a systematic review. Eur J Obstet Gynecol Reprod Biol 2013;170:1-7.

2. Maynard SE, Min JY, Merchan J, et al. Excess placental soluble fms-like tyrosine kinase 1 (sFlt1) may contribute to endothelial dysfunction, hypertension, and proteinuria in preeclampsia. J Clin Invest 2003;111:649-58

3. Allen RE, Rogozinska E, Cleverly K, et al. Abnormal blood biomarkers in early pregnancy are associated with preeclampsia: a metaanalysis. Eur J Obstet Gynecol Reprod Biol 2014;182:194-201.

4. Shore VH, Wang TH, Wang CL, et al. Vascular endothelial growth factor, placenta growth factor and their receptors in isolated human trophoblast. Placenta 1997;18:657-65.

5. Kusanovic JP, Romero R, Chaiworapongsa T, et al. A prospective cohort study of the value of maternal plasma concentrations of angiogenic and anti-angiogenic factors in early pregnancy and midtrimester in the identification of patients destined to develop preeclampsia. J Matern Fetal Neonatal Med 2009;22:1021-38.

6. Villa PM, Hämäläinen E, Mäki A, Pia MV, Esa H, Annukka M, et al. Vasoactive agents for the prediction of early- and late-onset preeclampsia in a high-risk cohort. BMC Pregnancy Childbirth 2013;13:110.

7. Verlohren S, Herraiz I, Lapaire O, et al. The sFIt-1/PIGF ratio in different types of hypertensive pregnancy disorders and its prognostic potential in preeclamptic patients. Am J Obstet Gynecol 2012;206:58.e1-8.

8. Levine RJ, Maynard SE, Qian C, et al. Circulating angiogenic factors and the risk of preeclampsia. N Engl J Med 2004;350:672-83.

9. Palm M, Basu S, Larsson A, et al. A longitudinal study of plasma levels of soluble fms-like tyrosine kinase 1 (sFlt1), placental growth factor (PIGF), sFlt1: PIGF ratio and vascular endothelial growth factor (VEGF-A) in normal pregnancy. Acta Obstet Gynecol Scand 2011;90:1244-51.

10. Stepan H, Herraiz I, Schlembach D, et al. Implementation of the sFIt-1/PIGF ratio for prediction and diagnosis of pre-eclampsia in singleton pregnancy: implications for clinical practice. Ultrasound Obstet Gynecol 2015;45:241-6.

11. Torry DS, Wang HS, Wang TH, et al. Preeclampsia is associated with reduced serum levels of placenta growth factor. Am J Obstet Gynecol 1998;179:1539-44.

12. Taylor RN, Grimwood J, Taylor RS, et al. Longitudinal serum concentrations of placental growth factor: evidence for abnormal placental angiogenesis in pathologic pregnancies. Am J Obstet Gynecol 2003;188:177-82.

13. Chappell LC, Seed PT, Briley A, et al. A longitudinal study of biochemical variables in women at risk of preeclampsia. Am J Obstet Gynecol 2002;187:127-36.

14. Powers RW, Roberts JM, Cooper KM, et al. Maternal serum soluble fms-like tyrosine kinase 1 concentrations are not increased in early pregnancy and decrease more slowly postpartum in women who develop preeclampsia. Am J Obstet Gynecol 2005;193:185-91.

15. Wolf $\mathrm{M}$, Shah $\mathrm{A}$, Lam $\mathrm{C}$, et al. Circulating levels of the antiangiogenic marker sFLT-1 are increased in first versus second pregnancies. Am J Obstet Gynecol 2005;193:16-22.

16. Khalil A, Maiz N, Garcia-Mandujano R, et al. Longitudinal changes in maternal serum placental growth factor and soluble fms-like tyrosine kinase-1 in women at increased risk of pre-eclampsia. Ultrasound Obstet Gynecol 2016;47:324-31.

17. Hirashima C, Ohkuchi A, Arai F, et al. Establishing reference values for both total soluble Fms-like tyrosine kinase 1 and free placental growth factor in pregnant women. Hypertens Res 2005;28:727-32.

18. Browne JL, Klipstein-Grobusch K, Koster MP, et al. Pregnancy associated plasma protein-a and placental growth factor in a subsaharan african population: a nested cross-sectional study. PLOS One 2016;11:e0159592. 